\title{
Optical Properties of Vanadium in $4 H$ Silicon Carbide for Quantum Technology
}

\author{
L. Spindlberger, ${ }^{1}$ A. Csóré, ${ }^{2}$ G. Thiering, ${ }^{2}$ S. Putz, ${ }^{3}$ R. Karhu, ${ }^{4}$ J. Ul Hassan, ${ }^{4}$ N.T. Son, ${ }^{4}$ \\ T. Fromherz, ${ }^{1}$ A. Gali, ${ }^{2,5}$ and M. Trupke ${ }^{3, *}$ \\ ${ }^{1}$ Institute of Semiconductor and Solid State Physics, Johannes Kepler University Linz, Altenberger Straße 69, \\ 4040 Linz, Austria \\ ${ }^{2}$ Institute for Solid State Physics and Optics, Wigner Research Center for Physics, Hungarian Academy of \\ Sciences, P.O. Box 49, Budapest, 1525, Hungary \\ ${ }^{3}$ Vienna Center for Quantum Science and Technology, Universität Wien, Boltzmanngasse 5, 1090 Vienna, Austria \\ ${ }^{4}$ Department of Physics, Chemistry and Biology, Linköping University, SE-581 83 Linköping, Sweden \\ ${ }^{5}$ Department of Atomic Physics, Budapest, University of Technology and Economics, Budafoki út 8 , \\ Budapest, 1111, Hungary
}

(Received 6 February 2019; revised manuscript received 17 April 2019; published 9 July 2019)

\begin{abstract}
We study the optical properties of tetravalent-vanadium impurities in $4 H$ silicon carbide. Light emission from two crystalline sites is observed at wavelengths of 1.28 and $1.33 \mu \mathrm{m}$, with optical lifetimes of 163 and $43 \mathrm{~ns}$, respectively, which remains stable up to 50 and $20 \mathrm{~K}$, respectively. Moreover, spectrally broad photoluminescence is observed up to room temperature. Group-theory and ab initio density-functional supercell calculations enable unequivocal site assignment and shed light on the spectral features of the defects. Specifically, our numerical simulations indicate that the site assignment is reversed with respect to previous assumptions. Our calculations show that vanadium in silicon carbide has highly favorable properties for the generation of single photons in the telecommunication wavelength regime. Combined with the available electronic and nuclear degrees of freedom, vanadium presents all the ingredients required for a highly efficient spin-photon interface.
\end{abstract}

DOI: 10.1103/PhysRevApplied.12.014015

\section{INTRODUCTION}

Defects in semiconductors such as silicon, diamond, and silicon carbide ( $\mathrm{SiC}$ ) have advanced to the forefront of candidates for the implementation of quantum bits and sensors as they have attractive features, such as long quantumcoherence lifetimes and strong optical transitions [1-15]. In diamond, nitrogen-vacancy defect has attracted particular interest due to its excellent room-temperature spin coherence, while the silicon-vacancy defect has a strong and narrow zero-phonon-line (ZPL) emission and only a weak phonon-assisted sideband. The luminescence of both defects appears in the visible portion of the spectrum. For long-distance communications and applications with optical interfaces, photonic signals in the near-infrared range between 1.2 and $1.6 \mu \mathrm{m}$ are most desirable, since scattering losses are greatly reduced and low absorption in silica optical fibers enables long-distance photon transmission. Furthermore, this wavelength range lies in the range of the biological transparency window.

$\mathrm{SiC}$ was recently discovered as a promising host for quantum bits and single-photon sources based on impurities [16]. Its large band gap (greater than $3 \mathrm{eV}$ ) gives

\footnotetext{
*michael.trupke@univie.ac
}

ample room for strong transitions in the optical domain, while both silicon and carbon are predominantly free of nuclear spin by natural isotopic abundance. The crystal therefore offers a quiescent magnetic environment even at natural isotopic abundance, and can be further enhanced by isotopic purification if needed [17]. Its crystal hardness gives rise to high-frequency phonon modes that are only modestly occupied, even at room temperature [18]. Recent study of optically active defects in silicon carbide revealed several defects with properties of interest for quantum applications [19]. Earlier work focused on the electronic properties of impurities, and included detailed research on metallic dopants, of which several possess optical transitions in the near-infrared region [20]. Detailed studies of these defects have only recently resumed in light of the emergence of quantum-enhanced applications. For instance, the chromium lines at 1042 and $1070 \mathrm{~nm}$ were examined recently, showing promise as a spin-photon interface [21]. However, the long optical lifetime indicated a modest optical cross section, presenting an obstacle for efficient photonic interactions.

Vanadium (V) is one of the most-scrutinized defects in $\mathrm{SiC}$ due to its amphoteric properties, which were routinely used for compensation of impure crystals. The first optical studies showed strong and sharp luminescence lines, as 
well as narrow ESR features with a linewidth smaller than $2 \mathrm{G}$ in an ensemble measured at temperatures of $77 \mathrm{~K}$ or higher [22]. This width corresponds to a spin coherence lifetime of several tens of nanoseconds, enabling coherent spin manipulation. The defect was further found to have multiple charge states, which are of interest for spin-to-charge conversion and nuclear-spin-memory protection [23-25]. This mechanism furthermore enables enhanced optical and even electrical read-out of the spin state by spin-selective ionization, as demonstrated recently for single nitrogen-vacancy centers in diamond [26,27].

These properties show promise for the application of vanadium in the emerging field of quantum technologies. With growing demand for efficient single-photon sources in the telecommunication wavelength regime, key for the implementation of quantum cryptography protocols or the generation of large entangled photon states, vanadium in silicon carbide is of great interest as it forms color centers emitting in the telecommunication wavelength regime. From a technological standpoint, electrical excitation $[28,29]$ of color centers is also desirable. Hence the $\mathrm{V}$ center in $\mathrm{SiC}$ creates the prospect of an electrically driven single-photon-emitting diode in a host that is a standard material in the semiconductor industry.

Moreover, the large hyperfine Hilbert space of the $\mathrm{V}$ centers' intrinsic nuclear spin points to the realization of solid-state quantum registers. Thus, vanadium in silicon carbide opens a route toward photonic spin interfaces, which allow scalable repeat-until-success quantumcomputing schemes and the generation of photonic cluster states [30-32]. However, many of the necessary characteristics of $\mathrm{V}$ in $\mathrm{SiC}$ remain to be determined, particularly regarding the details of their optical characteristics and their electronic structure.

In this work, we report on progress in this characterization by studying substitutional vanadium in silicon carbide with a view toward applications in quantum information and communication. We investigate the optical properties of the neutral charge state, $\mathrm{V}^{4+}$. This defect presents narrow optical emission lines in the telecommunication window near $1300 \mathrm{~nm}$, an optical decay lifetime of less than $50 \mathrm{~ns}$, and a rich level structure. The defect therefore shows promise for quantum photonics. We first describe its spectral features in Secs. II B and IIC. A theoretical picture of the defect properties is given in Sec. III. We conclude with an outlook for applications in photonic quantum technologies.

\section{EXPERIMENTAL RESULTS}

\section{A. Setup and materials}

We examine a single-crystal $4 H$-SiC sample with a homoepitaxial layer grown on an on-axis substrate [33]. The epilayer is $25 \mu \mathrm{m}$ thick and is grown with vanadium tetrachloride as a source of $\mathrm{V}$ dopant, leading to a V-inclusion density of $2 \times 10^{16} / \mathrm{cm}^{3}$ as measured by secondary-ion mass spectrometry. All photoluminescence measurements are performed with a $441-\mathrm{nm} \mathrm{cw}$ or pulsed laser source. The fluorescence is collected through the cryostat window with a $\times 100$ microscope objective (Mitutoyo M-Plan Apo, NA 0.7). Spectra are recorded with a grating spectrometer with a liquid-nitrogen-cooled detector, with a nominal resolution of $0.2 \mathrm{~nm}$ per pixel. All wavelengths are vacuum values. The fluorescence decay signals are recorded with a superconducting nanowire single-photon detector (EOS 210 OS from Single Quantum). The fluorescence signals from the different spectral features are isolated with use of narrow-bandpass filters with full widths at half maximum of approximately $15 \mathrm{~nm}$.

\section{B. Spectrum and polarization}

The spectrally resolved fluorescence [Fig. 1(a)] under blue excitation of the defects reveals three sharp lines, a doublet at wavelengths of 1278.0 and $1279.9 \mathrm{~nm}\left(\alpha_{3}\right.$ and $\alpha_{2}$ ) and a smaller feature at $1334 \mathrm{~nm}(\beta)$. The doublet has been attributed to the hexagonal site, and the single line has been attributed to the quasicubic site in $4 \mathrm{H}$-SiC. Our findings indicate that the correct assignment

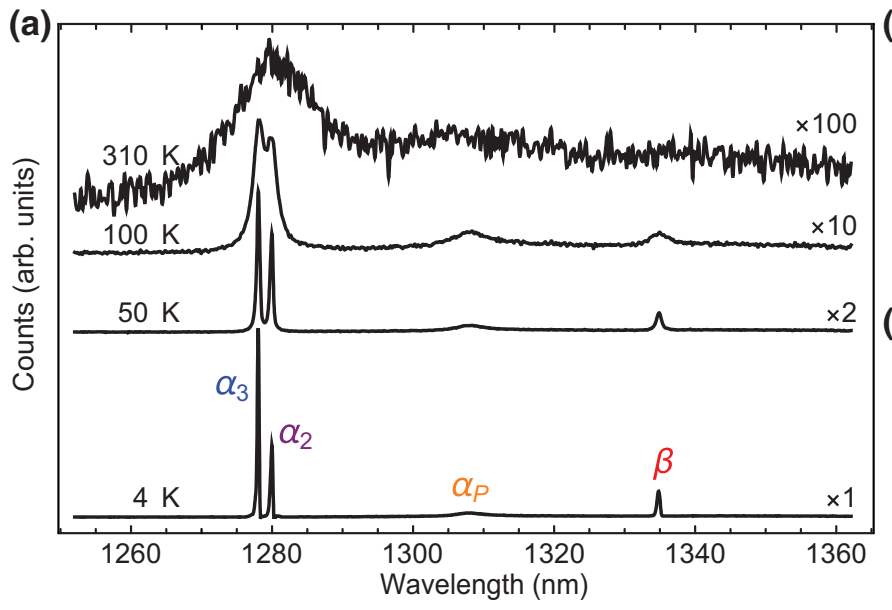

FIG. 1. (a) Recorded spectrum of the luminescence at selected temperatures showing a 2-nm split doublet associated with the hexagonal site. (b) Power dependence of the luminescence at $3.5 \mathrm{~K}$, showing no saturation in the measured regime. (c) Polarization dependence of the four different features (signals are rescaled for clarity, and the axes are with respect to the input field). 


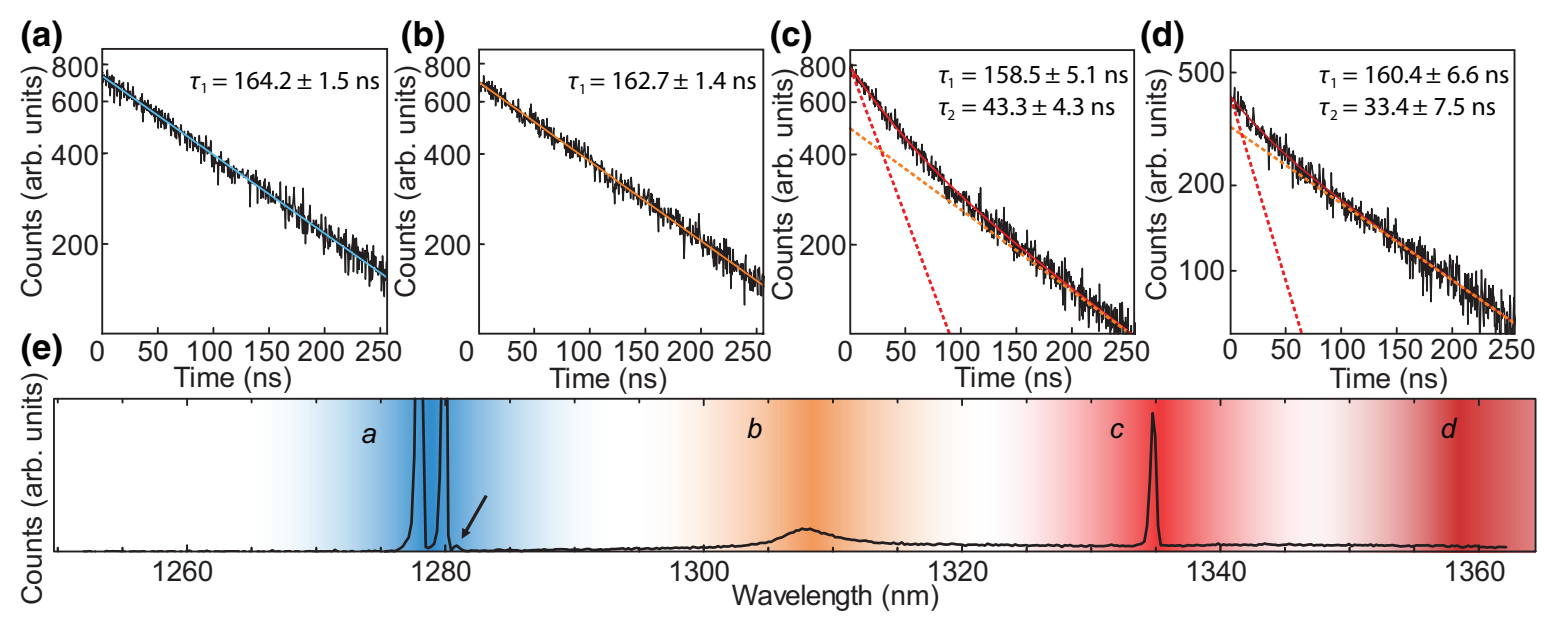

FIG. 2. Fluorescence decay for pulsed blue illumination measured at approximately 4 K. (a)-(d) Decay traces for filtered fluorescence in increasing wavelength ranges around 1280,1310, 1334, and $1360 \mathrm{~nm}$, respectively. The fitted decay functions are shown as solid lines. The dashed lines in (c),(d) display the individual fitted decay components. (e) Detail of the low-temperature spectrum. The four colored bands indicate the filtering bands for (a)-(d). The arrow indicates the spectral feature used for estimation of the spin-orbit splitting in the excited state.

is likely the opposite, as discussed in Sec. III. The broad feature between these lines near $1310 \mathrm{~nm}\left(\alpha_{P}\right)$ has not been assigned yet, but it has been hypothesized that it may correspond to a low-lying vibronic mode of the defect. The width of the $\alpha$ lines at temperatures below $30 \mathrm{~K}$ is below the spectrometer resolution (approximately $35 \mathrm{GHz}$ ) and is far smaller than their splitting of $355 \mathrm{GHz}$ for temperatures below $50 \mathrm{~K}$. We attribute the very weak feature near the strong $\alpha$ doublet, indicated in Fig. 2(e) by an arrow, to the $\alpha_{1}$ transition. Because of selection rules, this transition and a further feature at shorter wavelength are expected to be clearly observable only when the collection axis is perpendicular to the $c$ axis (see also Sec. II). The width of the $\beta$ feature approximately matches our spectrometer resolution. Previous measurements in $6 \mathrm{H}$-SiC, however, indicate that an unresolved doublet may give rise to the observed $\beta$ linewidth [34].

The doublet displays a temperature-dependent luminescence ratio. At $4 \mathrm{~K}$, the $\alpha_{3}$ peak accounts for almost $70 \%$ of the doublet's luminosity, but the ratio approaches equilibrium approximately exponentially and is near unity at $100 \mathrm{~K}$, above which temperature the lines are broadened beyond resolvability [Fig. 1(a)].

The polarization dependence of the luminescence on excitation power is consistent with a linear behavior within the available excitation range $(3.5 \mathrm{~mW}$ in front of the objective) [Fig. 1(b)]. We therefore expect that high luminosity can be achieved with significantly greater excitation power, or for resonant driving. The origin of the slight ellipticity in the emission is as yet unknown. Importantly, however, the polarization dependence of the luminescence displays near-identical behavior across the spectrum [Fig. 1(c)]. There are no indications for a strong spin dependence of the charge recapture, so it can be assumed that the excited-state spin sublevels are populated equally and with random phases in the excitation process.

The strength of the overall luminescence shows no discernible dependence on temperature up to $40 \mathrm{~K}$, and then decreases slowly by approximately $75 \%$ up to $310 \mathrm{~K}$.

\section{Fluorescence decay lifetimes}

The decay traces for the two types of defect illuminated with a pulsed blue laser are shown in Fig. 2. A portion of the trace before the laser pulse is used to calculate the background counts. The data for the $\alpha_{2}, \alpha_{3}$ doublet (black line) show very good agreement with a single-exponential decay (blue line) with lifetime $\tau_{1}=164.2 \pm 1.5 \mathrm{~ns}$, and a similar fluorescence lifetime is observed for the $\alpha_{P}$ feature. This observation confirms the assignment of the $\alpha_{P}$ feature to a low-lying phonon branch of the $\alpha$ defect. The $\beta$ line requires a double exponential,

$$
y(t)=A_{1} \exp \left(-t / \tau_{1}\right)+A_{2} \exp \left(-t / \tau_{2}\right),
$$

to reach agreement with the data. While the magnitudes $A_{1}$ and $A_{2}$ of the decay components are similar, the lifetimes differ significantly, with $\tau_{2}=158.5 \pm 5.1 \mathrm{~ns}$ and $\tau=43.3 \pm 4.3 \mathrm{~ns}$, where the error margins give the $3 \sigma$ uncertainty of the measured value. A similar behavior is observed in the spectral region around $1360 \mathrm{~nm}$. These characteristics indicate that local phonon modes give rise to a broad phonon sideband (PSB) that extends from the $\alpha$ ZPL peaks to beyond the $\beta$ feature. The doubleexponential behavior around the $\beta$ emission peak is then due to the addition of $\alpha$-related, phonon-assisted luminescence and $\beta$ ZPL photons, while at longer wavelengths it is caused by the addition of the phonon sidebands of the two defect types. 


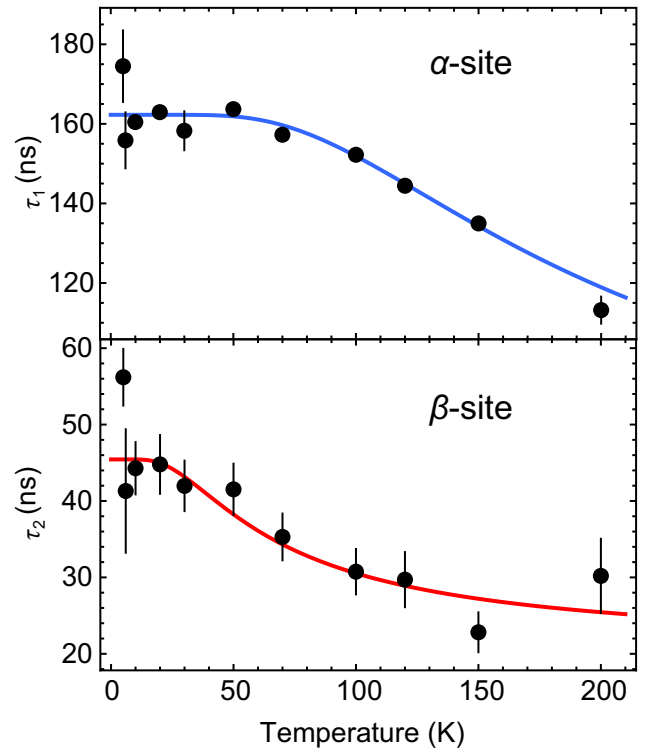

FIG. 3. Measured fluorescence decay lifetime versus temperature for the slower $(\alpha)$ and faster $(\beta)$ decay processes observed across the spectrum. The lines show fits to Eq. (2).

The decay rates show no statistically significant dependence on temperature up to $20 \mathrm{~K}$ for the $\beta$ decay channels and up to $50 \mathrm{~K}$ for the $\alpha$ lines. At higher temperatures, the luminescence decay accelerates significantly, as shown in Fig. 3. No spectral dependence of the decay lifetimes is discernible. To extract accurate values for the activation energy, we compound the decay rates extracted from decay traces recorded at around 1280,1334, and $1400 \mathrm{~nm}$. The data points thus show weighted averages of decay times extracted from traces at these points in the spectrum. We fit the observed decay rates to a model of thermally activated, nonradiative decay process, given by

$$
\tau_{\mathrm{tot}}(T)=\left[\frac{1}{\tau}+\frac{1}{\tau_{p}} \exp \left(-\frac{E_{p}}{k_{B} T}\right)\right]^{-1} .
$$

We find activation energies $E_{p}$ of $28 \pm 2 \mathrm{meV}$ and $8 \pm$ $3 \mathrm{meV}$ for the $\alpha$ and $\beta$ branches, with thermally assisted decay lifetimes $\tau_{p}$ of $83 \pm 15 \mathrm{~ns}$ and $36 \pm 11 \mathrm{~ns}$, respectively. These processes are likely related to thermally induced charge-transfer processes involving other impurities. Their relatively high activation energies nonetheless underline that both defects can be expected to display stable features at temperatures below $10 \mathrm{~K}$.

\section{THEORY AND NUMERICAL RESULTS}

\section{A. Defect model and details of calculations}

Previous results showed [35] that vanadium substitutes for the Si site in $4 H$-SiC. Since two inequivalent substitutional sites, $k$ and $h$, exist due to the particular packing of Si-C bilayers along the $c$ axis in $4 H-\mathrm{SiC}$, vanadium will form two distinct color centers in $4 H$-SiC. We label the two distinct configurations as vanadium $(k)$ and vanadium $(h)$ accordingly. We study the electronic structure and optical properties of these configurations by $a b$ initio calculations for identification of the vanadiumrelated $\alpha$ and $\beta$ emitters.

Calculations are performed in the framework of KohnSham density-functional theory (DFT) by means of the Perdew-Burke-Ernzerhof (PBE) [36] functional. We rationalize the choice of this functional in Sec. IIIB. The vanadium $(k)$ and $(h)$ defects are modeled in a 576-atom supercell. The Kohn-Sham wave functions are expressed by plane waves with a cutoff of $420 \mathrm{eV}$, where the ions are treated by projector-augmented-wave [37] potentials as implemented in VASP [38]. The large supercell suffices to apply a $\Gamma$ point for sampling the Brillouin zone, which also allows us to carefully follow the degeneracy of the defect levels in the fundamental band gap. The geometries are relaxed by our minimizing the total energy with respect to the coordinates of the atoms until the magnitude of the calculated forces was smaller than $0.01 \mathrm{eV} / \AA$.

The excited state is calculated by the delta-selfconsistent-field method [39] so as to determine the ZPL energy. To calculate the phonon sideband, the HuangRhys approximation is used as implemented by Gali et $a l$. [40]. The strength of the coupling is represented by the total Huang-Rhys factor $[S(\hbar \omega)]$. From $S(\hbar \omega)$, the spectral function $\left[A\left(E_{\mathrm{ZPL}}-\hbar \omega\right)\right]$ of the photolominescence spectrum can be calculated. The detailed derivation of $S(\hbar \omega)$ and $A\left(E_{\mathrm{ZPL}}-\hbar \omega\right)$ can be found in Refs. [40,41]. The contribution of the ZPL emission to the total emission containing the phonon sideband is the Debye-Waller $(W)$ factor, which can be read out from the experimental photoluminescence spectrum and is related to the calculated Huang-Rhys factor $S$ by $W=\exp [S(\hbar \omega)]$.

\section{B. Test on the applied semilocal DFT functional}

The HSE06 range-separated functional developed by Heyd, Scuseria, and Ernzerhof [42] was proven to be a powerful tool to study point defects in solids $[43,44]$. However, it has been shown that transition-metal defects can introduce strongly localized orbitals (e.g., $d$ electrons), which is significantly different from the character of the $s p^{3}$ electron bath of the host semiconductors, and HSE06 fails to simultaneously describe both states. To remedy this issue, Ivády et al. [45] developed a technique in which the HSE06 functional is corrected by an orbital-dependent term inspired by the DFT-with-the-Hubbard- $U$-parameter method that is called the "HSE06 $+V_{w}$ method." The additional term reads

$$
V_{w}=w\left(\frac{1}{2}-n\right)
$$

where $w$ represents the strength of the potential and $n$ is the occupation number. The value of $w$ can be determined via 


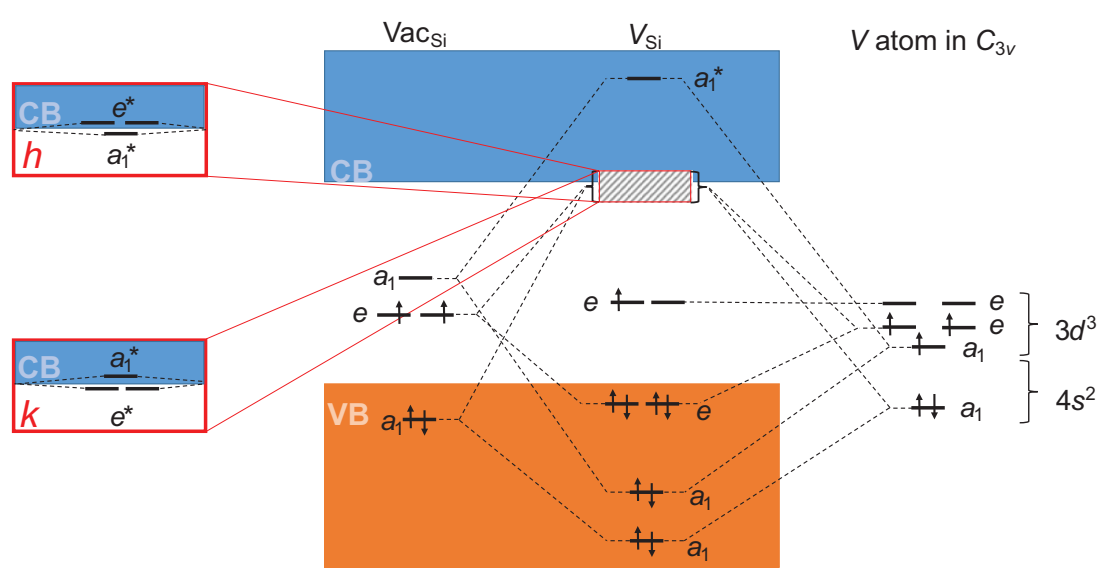

FIG. 4. Defect molecular-orbital diagram for vanadium $(h)$ and vanadium $(k)$ in $4 H$ $\mathrm{SiC}$ under $C_{3 v}$ symmetry regarding only the crystal-field splitting. Characters of all KohnSham levels are indicated. Antibonding levels are denoted by an asterisk. $\mathrm{Vac}_{\mathrm{Si}}$ indicates the silicon-vacancy levels. CB, conduction band; $\mathrm{VB}$, valence band.

the generalized Koopmans theorem [46]. We perform calculations to determine the potential strength, which yields $w=2.2 \mathrm{eV}$ for vanadium $(k)$ and vanadium $(h)$ defects, indicating identification of the accurate functional for the vanadium impurity in $4 H-\mathrm{SiC}$. We use this functional to test the computationally-less-costly semilocal PBE functional. We find that the order of defect levels for the two defect configurations agrees for the two methods (see Fig. 4). Furthermore, the localization of defect wave functions on vanadium atoms agrees within approximately $10 \%$ as obtained by the two methods. We conclude that the PBE functional can provide semiquantitative results for vanadium in $4 H-\mathrm{SiC}$, and thus we use this DFT functional in this context. However, this method is not expected to provide the correct absolute value for the energy of the excited state of the defects, for which we instead rely on the experimental data.

\section{Identification of emitters and calculated photoluminescence spectrum}

Vanadium is neutral in a wide range of doping levels [47]; thus, it is assumed that the $\alpha$ and $\beta$ emission lines originate from the neutral vanadium defects [48]. The observed spectral features support this model [20], which assumes a spin doublet ground state. We consider the neutral vanadium defect accordingly. Application of group theory can be very useful in determining the electronic structure of the defect. In Fig. 4 the defect molecularorbital diagram of the vanadium defect is depicted, which assumes an interaction between the Si-vacancy orbitals and vanadium atomic orbitals.

According to group-theory considerations the electronic structure introduced by the neutral vanadium defect is $a_{1}(2) a_{1}(2) e(4) e(1)$ whereas the empty states are $e^{*}$ and two $a_{1}^{*}$. The calculations imply that all the fully occupied states fall in the valence band and only the singly occupied atomiclike $e$ levels appear in the gap that constitutes the ${ }^{2} E$ ground state. Our DFT calculations reveal that the order of these $a_{1}^{*}$ and $e^{*}$ levels depends on the defect: $e^{*} a_{1}^{*}$ and $a_{1}^{*} e^{*}$ for the $k$ and $h$ sites, respectively (see Fig. 4).
To understand this effect, it is illustrative to derive the electronic structure from that of the vanadium defect in $3 C$ $\mathrm{SiC}$ exhibiting $T_{d}$ symmetry. Accordingly, vanadium atom introduces a doubly degenerate $e$ level in the band gap and a triply degenerate $t_{2}^{*}$ level resonant with the conductionband edge. Under the $C_{3 v}$ crystal field of the defect in $4 H$-SiC, the $t_{2}$ state splits into a doubly degenerate $e$ level and a nondegenerate $a_{1}$ level. The energy order of these states can be determined only by closer inspection of the defect environment [see Fig. 5(b)].According to the calculations, the $e^{*}$ state exhibits negligible extension along the $c$ axis, whereas the $a_{1}^{*}$ state $\left(d_{z z}\right.$-like orbital) is sensitive to the environment along the $c$ axis. Because of the crystal structure of $4 H-\mathrm{SiC}$, the $a_{1}$ state is more confined along the $z$-direction in the $(k)$ configuration than in the (h) configuration [dashed lines in Fig. 5(a)]. The stronger confinement at the $k$ site over the $h$ site pushes up the empty $a_{1}^{*}$ level for vanadium $(k)$ with respect to that for vanadium $(h)$, whereas the corresponding $e^{*}$ levels are the same.

These differences have considerable consequences for the nature of the photoluminescence from these defects. The emission comes from the ${ }^{2} E$ excited state at the $k$ site, whereas it comes from ${ }^{2} A_{1}$ at the $h$ site. This influences the fine structure of the excited state and the polarization of the emitted photons. Furthermore, the ZPL energy should be larger for the $(k)$ configuration than for the $(h)$ configuration if we assume the same Stokes shift in the emission process.

PBE DFT calculations result in ZPL energy of $0.845 \mathrm{eV}$ at the $k$ site and $0.785 \mathrm{eV}$ at the $h$ site. Here we ignored the spin-orbit splitting and used static Jahn-Teller distorted structures in the total-energy calculations. The absolute values of the calculated ZPL energies are within $0.1 \mathrm{eV}$. More importantly though, the calculated ZPL-energy difference with this simple method is $0.06 \mathrm{eV}$, which is close to the experimental value of $0.04 \mathrm{eV}$. These results strongly imply that $\alpha$ and $\beta$ emitters are the vanadium defects at the $k$ and $h$ sites, respectively, in contrast to the assumptions in the literature [22]. 
(a)

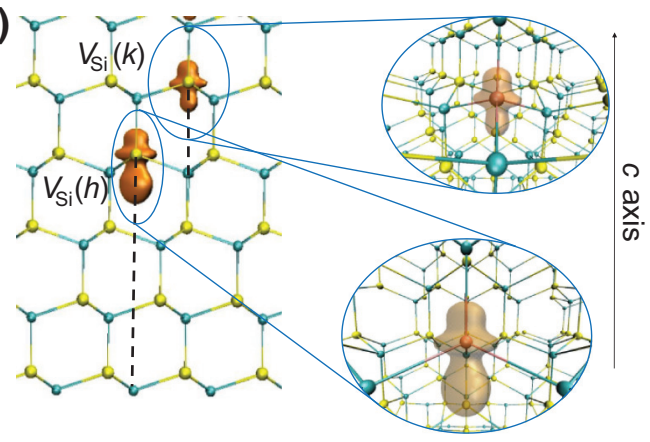

(b)

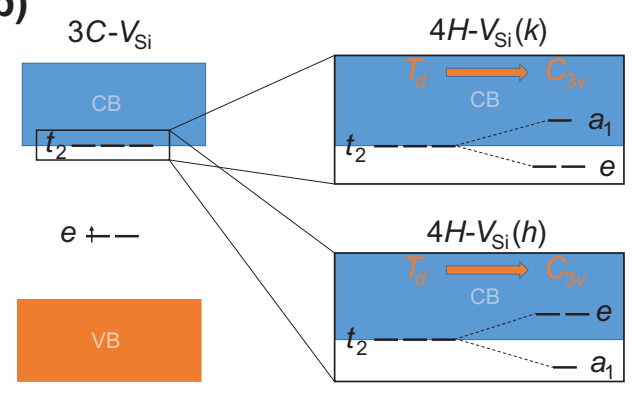

FIG. 5. (a) Defect models of vanadium $(h)$ and $(k)$ depicted in the same supercell in an orthographic view. Isosurfaces-using the same isovalue — of spin densities are illustrated for both defects. Dashed lines illustrate the distances of the nearest-neighbor atoms in the $z$ direction as the origin of the potential confinement along the $c$ axis. (b) Splitting of the $t_{2}$ level under $C_{3 v}$ symmetry. Both possible energy orderings of the splitting are depicted with the assignment of the corresponding vanadium defects in $4 H$-SiC. $\mathrm{CB}$, conduction band; VB, valence band.

Examination of the PSB of the vanadium emitters is of great importance as, on the one hand, it gives further insight into the optical properties of the defects and, on the other hand, it presents pathways for off-resonant excitation. The average $W$ factor of the two defects can be evaluated directly from the measurement and is $34 \%$. We can further calculate limits on the $W$ factors of the two defects by partitioning the spectrum at the $\beta$ PSB, which yields $31 \%<W_{\alpha}<60 \%$ and $W_{\beta}>10 \%$. To gain further understanding of the phononic properties of the two emitters, we construct a numerically optimized spectrum to match the observed photoluminescence (see Fig. 6). Guided by the $a b$ initio model, we assume that the $\beta$ defect has no significant PSB emission with phonon energies less than approximately $20 \mathrm{meV}$. We furthermore impose the condition that the PSB emission is zero for phonon energies greater than $200 \mathrm{meV}$. We then construct a summed spectrum from five Gaussian sidebands of the form

$$
I(\delta)=I_{0} \sum_{j=1}^{j=10} \frac{1}{\sqrt{j \pi} \sigma} \exp \left[-\left(\frac{\delta-\Delta_{0}}{\sqrt{j} \sigma}\right)^{2}\right] .
$$

Each sideband is then turned into a weighted doublet to account for the $\alpha_{2}$ and $\alpha_{3}$ emission by appropriate adjustment of the factors $\Delta_{0}$ and $I_{0}$ to match the observed $1.47-\mathrm{meV}$ splitting and strengths in the $\alpha$ ZPL. The remaining PSB luminescence is mostly assigned to the $\beta$ defect, aside from a prominent set of features near the $\alpha$ phonon-energy band. A summary of the assigned photoluminescence features in Fig. 6 is shown in Table I. The calculated spectrum allows us to refine our estimates to $W_{\alpha} \sim 39 \%$ and $W_{\beta} \sim 22 \%$.

Table II lists the calculated Huang-Rhys $S$ and the derived $W$ factors, as well as the calculated radiative lifetime $\tau_{\text {rad }}$. Comparison of the calculated radiative lifetime with the measured photoluminescence lifetime yields an estimate of the radiative efficiency $\eta_{\text {rad }}$. A lower limit for the total efficiency of the ZPL transition, $\eta_{\text {tot }}$, is then calculated as the product of $\eta_{\mathrm{rad}}$ and the estimated $W$ factors.

The difference in the experimentally deduced and theoretical $W$ factors may arise from the sharp phonon peaks in the observed photoluminescence spectra, which are not reproduced by our simulations. These cannot be accounted for by Huang-Rhys theory, and may be due to an HerzbergTeller mechanism [49]. In particular, the very sharp $\beta$ feature marked $\Sigma_{\beta}$ in Fig. 6 cannot be assigned to a

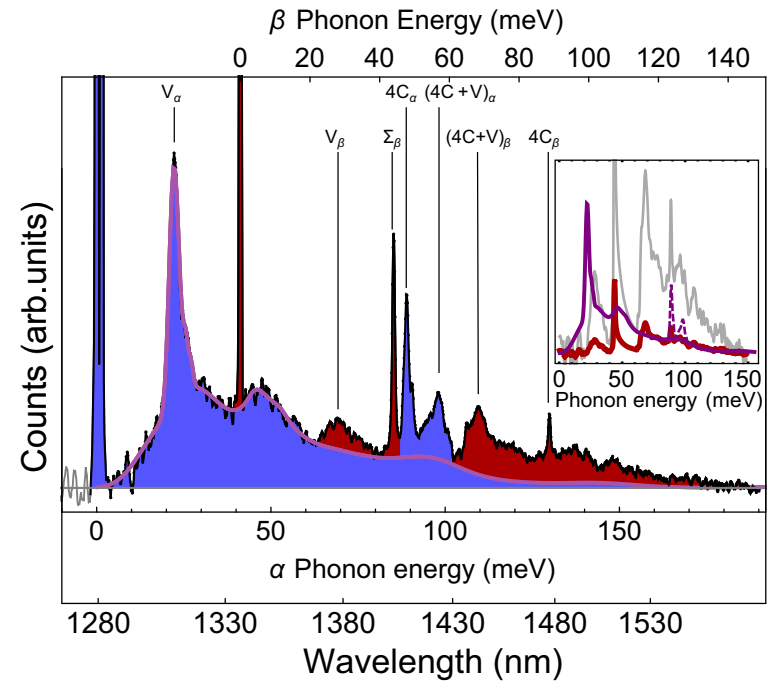

FIG. 6. Detail of the phonon sideband, with assignments extracted from the $a b$ initio defect models. The purple line shows a fit to the features with energy below $64 \mathrm{meV}$ that are assumed to originate solely from the $\alpha$ defect. The blue (red) shaded area is assigned to the $\alpha(\beta)$ line. The inset shows the fitted $\alpha$ phonon energy (purple line) compared with the extrapolated and shifted $\beta$ features (red line, unscaled; gray line, scaled to equal ZPL intensity). The lines are smoothed and interpolated for clarity. The dashed segment is the overlayed $4 \mathrm{C}_{\alpha}$ and $(4 \mathrm{C}+\mathrm{V})_{\alpha}$ portion of the recorded PSB. 
TABLE I. Summary of the observed photoluminescence features with phonon energy and corresponding absorption wavelengths according to the defect assignment shown in Fig. 6 . The calculated absorption-feature wavelengths are given in the column " $\lambda_{\text {abs }}$ (calc.)."

\begin{tabular}{lccccc}
\hline \hline$\alpha$ phonon & Energy $(\mathrm{meV})$ & $\lambda_{\text {abs }}($ calc. $)(\mathrm{nm})$ & $\beta$ phonon & Energy $(\mathrm{meV})$ & $\lambda_{\text {abs }}(\mathrm{calc}).(\mathrm{nm})$ \\
\hline $\mathrm{V}_{\alpha}$ & 22 & 1252 & $\mathrm{~V}_{\beta}$ & 29 & 1295 \\
$4 \mathrm{C}_{\alpha}$ & 89 & 1173 & $\Sigma_{\beta}$ & 45 & 1275 \\
$(4 \mathrm{C}+\mathrm{V})_{\alpha}$ & 98 & 1163 & $(4 \mathrm{C}+\mathrm{V})_{\beta}$ & 69 & 1244 \\
& & $4 \mathrm{C}_{\beta}$ & 89 & 1219 \\
\hline \hline
\end{tabular}

particular oscillatory mode. This feature, however, has a strong absorption cross section far from resonance $(\lambda=$ $1275 \mathrm{~nm})$ from the $\beta$ ZPL (1334 nm), which will be of benefit for photon-source applications. Similarly, the $4 \mathrm{C}_{\alpha}$ feature is expected to have a corresponding absorption feature near $1173 \mathrm{~nm}$, and may even enable state-selective driving of the defect since the $\alpha_{2}$ and $\alpha_{3}$ components are at least partially resolved. As the energy gap is smaller in the $(h)$ configuration than in the $(k)$ configuration, the nonradiative lifetime of the $(h)$ configuration can be expected to be shorter. Its radiative lifetime is also shorter than that of the $(k)$ configuration. It is conceivable that the moredelocalized excited wave function is responsible for this feature. We furthermore note that the use of blue excitation may lead to nonradiative recombination pathways that are excluded when resonant excitation is used. It is therefore possible that a photoluminescence-excitation experiment may reveal longer excited-state lifetimes than those observed here, yielding higher transition efficiencies.

\section{Quantum optical properties}

While V in SiC shares some features with the silicon vacancy in diamond [6,7], there are important differences beyond the beneficial optical frequency. As shown in Figs. 7(a) and 7(b), the spin-orbit (SO) splitting for the hexagonal $(h)$ site is comparable to the silicon-vacancy SO splitting in diamond, while the cubic $(k)$ site shows a ground-state SO splitting which is more than ten times larger [50]. The ground state is seated deeply within the band gap, enabling strong localization of the electron wave function. This points to long spin coherence lifetimes, which have indeed been observed in ensembles [22]. The $\alpha$ defect at the cubic site is particularly promising for spin-based applications as its large ground-state splitting will likely permit longer coherence times at moderate cryogenic temperatures. Furthermore, together with the relatively large nuclear magnetic moment of ${ }^{51} \mathrm{~V}(S=$ $\left.7 / 2, g_{N}=1.471 \mu_{N}\right)$, the strong localization of the electron wave function enables a significantly larger hyperfine coupling frequency $\left(A_{\|}=235 \mathrm{MHz}\right)$ than the ESR linewidth $[20,51]$. The hyperfine octet furthermore offers a large Hilbert space for quantum-information storage and manipulation [52,53]. Very recently demonstrated protocols for nuclear spin control with the silicon-vacancy color center in diamond indicate that defects of this type can serve as an interface for long-lived nuclear-spin qubits [54].

An important application for these defects will be the interaction with photons, which can be enhanced in a photonic crystal $[55,56]$ in $\mathrm{SiC}$, or in a Fabry-Perot optical resonator. In such devices the photon collection can be strongly enhanced. Furthermore, spin read-out by statedependent reflectivity can reach high fidelity and can be made nondestructive. Silicon carbide membranes have been shown to retain extremely high strength even for submicrometer thickness [57]. Furthermore, it has been shown that the $\mathrm{SiC}$ surface can be polished to subangstrom roughness [58]. From our measurements we estimate $\eta_{\text {tot }} \sim$ $8.9 \%$ for the transition efficiency of the $\alpha$ doublet, and thus we can calculate the cooperativity that can be achieved in a Fabry-Perot resonator:

$$
C=\frac{2}{\pi} \frac{\sigma_{E}}{\sigma_{C}} \eta_{\mathrm{tot}} \frac{f_{L}}{n_{\mathrm{SiC}}} F
$$

which is proportional to the relation of the optical cross sections of the emitter $\sigma_{E}$ and the beam waist $\sigma_{C}$. The factor $f_{L}=\left(L_{\mathrm{vac}}+n_{\mathrm{SiC}} L_{\mathrm{SiC}}\right) /\left(L_{\mathrm{vac}}+n_{\mathrm{SiC}}^{2} L_{\mathrm{SiC}}\right)$ accounts for the modification of the electric field strength in a heterogeneous cavity partially filled with $\mathrm{SiC}$ with refractive index $n_{\mathrm{SiC}}$ and path length $L_{\mathrm{SiC}}$. The refractive index of the

TABLE II. Summary of measured and calculated radiative properties for the $(h)$ and $(k)$ configurations of $\mathrm{V}^{4+}$ in $4 H$-SiC. (th.) and (expt.) indicate calculated and measured values, respectively. $\tau_{\mathrm{NR}}, \eta_{\mathrm{rad}}$, and $\eta_{\mathrm{tot}}$ are extracted from the preceding measured and calculated values.

\begin{tabular}{lcccccccc}
\hline \hline Site & $S$ (th.) & $W$ factor (th.) & $W$ factor & $\tau_{\text {rad }}($ th. $)(\mathrm{ns})$ & $\tau_{\text {tot }}($ expt.) $(\mathrm{ns})$ & $\tau_{\mathrm{NR}}(\mathrm{ns})$ & $\eta_{\text {rad }}(\%)$ & $\eta_{\text {tot }}(\%)$ \\
\hline$k$ & 0.66 & 0.52 & 0.39 & 704 & $\tau_{1}=163$ & 212 & 23 \\
$h$ & 0.79 & 0.45 & 0.22 & 277 & $\tau_{2}=43$ & 47 & 15 & 3.3 \\
\hline \hline
\end{tabular}



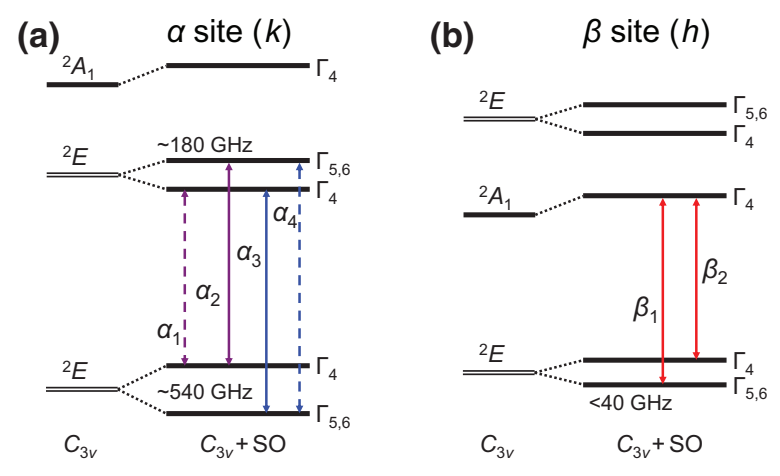

FIG. 7. Level structure with tentative assignment from group theory, simulation, previous work [50], and measurement. (a) $\alpha$ lines originating from the cubic $(k)$ site. The weak-luminescence feature at slightly lower energy than the $\alpha_{2}, \alpha_{3}$ doublet allows us to estimate the SO splittings as shown (see Fig. 2). Dashed and straight arrows represent the polarization of the photons parallel to the $c$ axis ( $\pi$ or $z$ transition) and perpendicular to the $c$ axis ( $\sigma$ or $x-y$ plane), respectively, in the optical transition. (b) $\beta$ lines originating from the hexagonal $(h)$ site.

vacuum air is set to 1 , and the corresponding path length is denoted by $L_{\mathrm{vac}}$. The term is found by integrating over the mode volume [59] of the optical cavity, assuming an antireflection (AR) coating on the inner surface and small beam expansion. The cavity finesse $F$ in Eq. (5) is proportional to the photon round-trip number, and hence accounts for the enhancement due to the optical resonator.

Here $\sigma_{E}=3 \lambda^{2} / 2 \pi$ is the ideal optical cross section of the emitter, while $\sigma_{C}=\pi w_{C}^{2}$ is the cross section of the cavity mode with the $1 / e^{2}$ intensity radius $w_{C}=$ $\sqrt{\lambda / \pi}\left[L_{d}\left(R_{C}-L_{d}\right)\right]^{1 / 4}$ in the paraxial approximation. $L_{d}=L_{\mathrm{vac}}+L_{\mathrm{SiC}} / n_{\mathrm{SiC}}$ is the effective path length for the Gaussian beam, once again under the approximation of paraxial propagation and a thin $\mathrm{SiC}$ layer compared with the Rayleigh length. The field within the AR coating is neglected. We choose $L_{\mathrm{vac}}=1.05 \mu \mathrm{m}$ and $L_{\mathrm{SiC}}=0.6 \mu \mathrm{m}$. Silicon microcavities provide an ideal platform for this endeavor given their scalability, supreme surface quality, and transparency in the telecommunication band [60-62].

The finesse for such a device is given by $F=2 \pi /\left(T_{1}+\right.$ $\left.\ell_{\text {tot }}\right)$, where $\ell_{\text {tot }}=A_{1}+T_{2}+A_{2}+S_{2}+\ell_{\sigma}$ contains all undesired loss channels, including the absorption losses in the output mirror $A_{1}$ and the transmission, scattering, and absorption of the second mirror $T_{2}, A_{2}$, and $S_{2}$. The losses in the cavity will most likely be dominated by scattering at the $\mathrm{SiC}$ surfaces, $\ell_{\sigma} \simeq\left(4 \pi n_{\mathrm{SiC}} \sigma / \lambda\right)^{2}+$ $2\left[\left(n_{\mathrm{SiC}}-1\right) 2 \pi \sigma / \lambda\right]^{2}$, where the two terms give the loss at the external mirror and at the internal interface, respectively. Assuming a roughness of $\sigma=0.1 \mathrm{~nm}$ on both faces of the $\mathrm{SiC}$ membrane, $\ell_{\sigma}=7.8 \mathrm{ppm}$. This value allows us to calculate the achievable cooperativity $C$ and Purcell factor $P=2 C+1$. With current, published values $\left(169-\mu \mathrm{m}\right.$ radius of curvature, $T_{1}=T_{2}=5 \mathrm{ppm}, A_{1}=$ $A_{2}=1 \mathrm{ppm}, S_{2}=2 \mathrm{ppm}$ [63]), we find an attainable cooperativity $C$ of 134 . This value is promising for optically interfaced qubit networks [30] since it delivers nearunity cavity reflection for quantum-state read-out [64], even when the cavity contrast is maximized $\left(T_{1} \rightarrow \ell_{\text {tot }}\right.$, $C=87$ ). This cooperativity furthermore corresponds to a significant reduction of the excited-state lifetime to $6.5 \mathrm{~ns}$. The value is calculated from $1 / \tau_{\text {tot }}=P / \tau_{\mathrm{ZPL}}+1 / \tau_{\mathrm{PSB}}+$ $1 / \tau_{\mathrm{NR}}$, where $\tau_{\mathrm{ZPL}}=\tau_{\mathrm{rad}} / W$ and $\tau_{\mathrm{PSB}}=\tau_{\mathrm{rad}} /(1-W)$. The Purcell-enhanced relaxation into the cavity on the ZPL is then given by $\eta_{P}=2 C /\left(2 C+1 / \eta_{\text {tot }}\right)$, while the fraction of these photons that exit the output mirror is $\eta_{T}=T_{1} /\left(T_{1}+\ell_{\text {tot }}\right)$.

The peak brightness of such a source is given by maximizing $2 C \eta_{T}$ by choosing $T_{1} \rightarrow \ell_{\text {tot }}$, where the excitedstate lifetime is $9.8 \mathrm{~ns}$ and the output efficiency (output probability per excitation) is $47 \%$. Repetition rates on the order of tens of megahertz are therefore possible while maintaining good efficiency. The highest output efficiency of the coupled emitter-cavity system is instead found by maximizing the product $\eta_{P} \times \eta_{T}$, and is $\eta_{\max }=(1-\sqrt{2 x+1}) / x$, with $x=2 \pi\left(\eta_{\text {tot }} / \ell_{\text {tot }}\right)(C / F)$. For high efficiency, this simplifies to $1-\sqrt{2 / x}=1-$ $\left(\pi w_{C} / \eta_{\text {tot }} \lambda\right) \sqrt{\ell_{\text {tot }} n_{\mathrm{SiC}} / 3 f_{L}}$, which underlines the advantage of a longer wavelength and the importance of the mode waist and branching ratio. Optimizing the output transmission for photon-collection efficiency with $T_{1}(\mathrm{opt})=$ $\sqrt{\ell_{\text {tot }}^{2}+4 \pi \ell_{\text {tot }} \eta_{\text {tot }} C / F}$ yields an emission probability per excitation of $70 \%$, while maintaining a comparatively short excited-state lifetime of 28.5 ns. A further reduction of the mirror dimensions [65-68] could conceivably lead to output-efficiency values exceeding $90 \%$. These values indicate that $\mathrm{V}$ in $\mathrm{SiC}$ may serve as a fast and highly efficient cavity-enhanced single-photon source, with performance at or beyond the state of the art [69]. The availability of spin degrees of freedom furthermore provides a path toward the generation of entangled photon states for quantum computation and communication at a wavelength compatible with silicon photonics and long-haul optical fiber networks $[32,70]$.

\section{DISCUSSION}

The optical properties of the vanadium defects studied indicate that they are strong candidates for photonic applications. The recorded spectrum is in agreement with the literature. However, our $a b$ initio simulations suggest that the site assignment of the two defects should be reversed: the $\alpha$ lines originate from the cubic site, while the $\beta$ lines are emitted from the hexagonal site. The fluorescence decay is remarkably fast for an intrashell $3 d$ transition.

The double-exponential decay recorded in the spectral region of the $\beta$ line most likely results from the sum of phonon-assisted $\alpha$ luminescence and a faster optical transition in the $\beta$ defect. Taken together, the shorter lifetime and the lower fluorescence count rate could be caused by 
stronger nonradiative or phonon-assisted decay processes for the $\beta$ defect, but the lower count rate could also be due to a reduced recombination efficiency in the excited state, since the electron wave function is more strongly localized at this site.

Future theoretical efforts should be on deepening our understanding of the spin-orbit structure and of the possible Herzberg-Teller features in the PSB. Resonant spectroscopy will be required to shed further light on the nature of the decay processes, including nonradiative decay channels and thermally enhanced decay rates.

Both sites present a rich level structure that will enable coherent optical manipulation and spin-selective optical transitions, in turn leading to applications in quantum communication and computation [30,71-73]. These mechanisms can be further enhanced by placing the defects into optical microcavities, where the long wavelength will be beneficial in reducing losses due to scattering. Furthermore, the transitions both lie in the transparency window of silicon, opening the possibility of integration with photonic, mechanical, and electronic structures in the mostdeveloped semiconductor platform $[61,74]$. Furthermore, the emission lies in the second biological window, and may therefore be of interest for in vivo bioimaging.

In summary, we study the luminescence of neutral substitutional vanadium centers in $4 H$-SiC. Temperatureand time-dependent measurements allow us to shed light on previously unassigned features of the spectrum, and reveal attractive features, including a small inhomogeneous linewidth and fast optical transitions. The characteristics of the vanadium centers studied herein are reminiscent of the molybdenum defect in $\mathrm{SiC}$ and the silicon-vacancy complex in diamond [6,7,12], but at technologically practical wavelengths in the telecommunication $\mathrm{O}$ band, and favorable spin properties. The centers therefore merit further examination for applications in quantum technology.

\section{ACKNOWLEDGMENTS}

Financial support was provided by the FWF project I 3167-N27 SiC-EiC, the OeAD project HU 12/2016 PERFEQT, the Carl-Tryggers Stiftelse för Vetenskaplig Forskning (Grant No. CTS 15:339), the Swedish Research Council (Grant No. VR 2016-04068), the Swedish Energy Agency (Grant No. 43611-1), and the Knut and Alice Wallenberg Foundation (Grant No. KAW 2018.0071). Support from the ÚNKP-18-3-I New National Excellence Program of the Ministry of Human Capacities of Hungary is acknowledged by A.C. A.G. acknowledges support from the National Research, Development and Innovation Office in Hungary (NKFIH) under Grants No. 2017-1.2.1-NKP-2017-00001 (National Quantum Technology Program), No. NVKP 16-1-2016-0043 (NVKP Program), No. NN127902 (EU QuantERA Nanospin
Project), and No. KKP129866 (National Excellence Program: Quantum-Coherent Materials Project).

L.S., T.F., and M.T. performed the measurements. A.C., G.T., and A.G. simulated the defect structure and performed group-theory calculations. R.K. and J.U.H. produced the sample. M.T. initiated and coordinated the work, and wrote the manuscript. All authors contributed to data analysis and to the manuscript text.

[1] M. W. Doherty, N. B. Manson, P. Delaney, and F. Jelezko, The nitrogen-vacancy colour centre in diamond, Phys. Rep. 528, 1 (2013).

[2] L. Rondin, J. P. Tetienne, T. Hingant, J. F. Roch, P. Maletinsky, and V. Jacques, Magnetometry with nitrogenvacancy defects in diamond, Rep. Prog. Phys. 77, 056503 (2014).

[3] G. Kucsko, P. C. Maurer, N. Y. Yao, M. Kubo, H. J. Noh, P. K. Lo, H. Park, and M. D. Lukin, Nanometre-scale thermometry in a living cell, Nature 500, 54 (2013).

[4] F. Dolde, H. Fedder, M. W. Doherty, T. Nöbauer, F. Rempp, G. Balasubramanian, T. Wolf, F. Reinhard, L. C. L. Hollenberg, F. Jelezko, and J. Wrachtrup, Electric-field sensing using single diamond spins, Nat. Phys. 7, 459 (2011).

[5] P. Neumann, I. Jakobi, F. Dolde, C. Burk, R. Reuter, G. Waldherr, J. Honert, T. Wolf, A. Brunner, J. H. Shim, D. Suter, H. Sumiya, J. Isoya, and J. Wrachtrup, High-precision nanoscale temperature sensing using single defects in diamond, Nano Lett. 13, 2738 (2013).

[6] L. J. Rogers, K. D. Jahnke, M. W. Doherty, A. Dietrich, L. P. McGuinness, C. Müller, T. Teraji, H. Sumiya, J. Isoya, N. B. Manson, and F. Jelezko, Electronic structure of the negatively charged silicon-vacancy center in diamond, Phys. Rev. B 89, 235101 (2014).

[7] J. N. Becker, J. Görlitz, C. Arend, M. Markham, and C. Becher, Ultrafast all-optical coherent control of single silicon vacancy colour centres in diamond, Nat. Commun. 7, 13512 (2016).

[8] A. Sipahigil, R. E. Evans, D. D. Sukachev, M. J. Burek, J. Borregaard, M. K. Bhaskar, C. T. Nguyen, J. L. Pacheco, H. A. Atikian, C. Meuwly, R. M. Camacho, F. Jelezko, E. Bielejec, H. Park, M. Lončar, and M. D. Lukin, An integrated diamond nanophotonics platform for quantumoptical networks, Science 354, 847 (2016).

[9] D. J. Christle, A. L. Falk, P. Andrich, P. V. Klimov, J. Ul Hassan, N. T. Son, E. Janzén, T. Ohshima, and D. D. Awschalom, Isolated electron spins in silicon carbide with millisecond coherence times, Nat. Mater. 14, 160 (2015).

[10] M. Widmann, S.-Y. Lee, T. Rendler, N. T. Son, H. Fedder, S. Paik, L.-P. Yang, N. Zhao, S. Yang, I. Booker, A. Denisenko, M. Jamali, S. Ali Momenzadeh, I. Gerhardt, T. Ohshima, A. Gali, E. Janzén, and J. Wrachtrup, Coherent control of single spins in silicon carbide at room temperature, Nat. Mater. 14, 164 (2015).

[11] F. Fuchs, B. Stender, M. Trupke, D. Simin, J. Pflaum, V. Dyakonov, and G. V. Astakhov, Engineering near-infrared single-photon emitters with optically active spins in ultrapure silicon carbide, Nat. Commun. 6, 7578 (2015). 
[12] T. Bosma, G. J. J. Lof, C. M. Gilardoni, O. V. Zwier, F. Hendriks, A. Gällström, I. G. Ivanov, N. T. Son, R. W. A. Havenith, and C. H. van der Wal, Identification and tunable optical coherent control of transition-metal spins in silicon carbide, npj Quantum Inf. 4, 48 (2018).

[13] M. Atatüre, D. Englund, N. Vamivakas, S.-Y. Lee, and J. Wrachtrup, Material platforms for spin-based photonic quantum technologies, Nat. Rev. Mater. 3, 38 (2018).

[14] K. J. Morse, R. J. S. Abraham, A. DeAbreu, C. Bowness, T. S. Richards, H. Riemann, N. V. Abrosimov, P. Becker, H.-J. Pohl, M. L. W. Thewalt, and S. Simmons, A photonic platform for donor spin qubits in silicon, Sci. Adv. 3, e1700930 (2017).

[15] C. Beaufils, W. Redjem, E. Rousseau, V. Jacques, A. Yu. Kuznetsov, C. Raynaud, C. Voisin, A. Benali, T. Herzig, S. Pezzagna, J. Meijer, M. Abbarchi, and G. Cassabois, Optical properties of an ensemble of g-centers in silicon, Phys. Rev. B 97, 035303 (2018).

[16] S. Castelletto, B. C. Johnson, V. Ivády, N. Stavrias, T. Umeda, A. Gali, and T. Ohshima, A silicon carbide roomtemperature single-photon source, Nat. Mater. 13, 151 (2013).

[17] D. Simin, V. A. Soltamov, A. V. Poshakinskiy, A. N. Anisimov, R. A. Babunts, D. O. Tolmachev, E. N. Mokhov, M. Trupke, S. A. Tarasenko, A. Sperlich, P. G. Baranov, V. Dyakonov, and G. V. Astakhov, All-Optical DC Nanotesla Magnetometry Using Silicon Vacancy Fine Structure in Isotopically Purified Silicon Carbide, Phys. Rev. X 6, 031014 (2016).

[18] T. Egilsson, J. P. Bergman, I. G. Ivanov, A. Henry, and E. Janzén, Properties of the D1 bound exciton in $4 \mathrm{H}-\mathrm{SiC}$, Phys. Rev. B 59, 1956 (1999).

[19] J. R. Weber, W. F. Koehl, J. B. Varley, A. Janotti, B. B. Buckley, C. G. Van de Walle, and David D. Awschalom, Quantum computing with defects, Proc. Natl. Acad. Sci. U. S. A. 107, 8513 (2010).

[20] J. Baur, M. Kunzer, and J. Schneider, Transition metals in $\mathrm{SiC}$ polytypes, as studied by magnetic resonance techniques, Phys. Status Solidi (a) 162, 153 (1997).

[21] W. F. Koehl, B. Diler, S. J. Whiteley, A. Bourassa, N. Tien Son, E. Janzén, and D. D. Awschalom, Resonant optical spectroscopy and coherent control of $\mathrm{Cr}^{4+}$ spin ensembles in $\mathrm{SiC}$ and GaN, Phys. Rev. B 95, 035207 (2017).

[22] J. Schneider, H. D. Müller, K. Maier, W. Wilkening, F. Fuchs, A. Dörnen, S. Leibenzeder, and R. Stein, Infrared spectra and electron spin resonance of vanadium deep level impurities in silicon carbide, Appl. Phys. Lett. 56, 1184 (1990).

[23] J. M. Elzerman, R. Hanson, L. H. Willems Van Beveren, B. Witkamp, L. M. K. Vandersypen, and L. P. Kouwenhoven, Single-shot read-out of an individual electron spin in a quantum dot, Nature 430, 431 (2004).

[24] K. Saeedi, S. Simmons, J. Z. Salvail, P. Dluhy, H. Riemann, N. V. Abrosimov, P. Becker, H.-J. Pohl, J. J. L. Morton, and M. L. W. Thewalt, Room-temperature quantum bit storage exceeding 39 minutes using ionized donors in silicon-28, Science 342, 830 (2013).

[25] M. Pfender et al., Protecting a diamond quantum memory by charge state control, Nano Lett. 17, 5931 (2017).

[26] B. J. Shields, Q. P. Unterreithmeier, N. P. de Leon, H. Park, and M. D. Lukin, Efficient Readout of a Single Spin State in
Diamond via Spin-to-Charge Conversion, Phys. Rev. Lett. 114, 136402 (2015).

[27] P. Siyushev, M. Nesladek, E. Bourgeois, M. Gulka, J. Hruby, T. Yamamoto, M. Trupke, T. Teraji, J. Isoya, and F. Jelezko, Photoelectrical imaging and coherent spin-state readout of single nitrogen-vacancy centers in diamond, Science 363, 728 (2019).

[28] F. Hargart, C. A. Kessler, T. Schwarzbäck, E. Koroknay, S. Weidenfeld, M. Jetter, and P. Michler, Electrically Driven Quantum Dot Single-Photon Source at $2 \mathrm{GHz}$ Excitation Repetition Rate with Ultra-Low Emission Time Jitter, Appl. Phys. Lett. 102, 011126 (2013).

[29] A. Lohrmann, N. Iwamoto, Z. Bodrog, S. Castelletto, T. Ohshima, T. J. Karle, A. Gali, S. Prawer, J. C. McCallum, and B. C. Johnson, Single-photon emitting diode in silicon carbide, Nat. Commun. 6, 7783 (2015).

[30] K. Nemoto, M. Trupke, S. J. Devitt, A. M. Stephens, B. Scharfenberger, K. Buczak, T. Nöbauer, and M. S. Everitt, J. Schmiedmayer, and W. J. Munro, Photonic Architecture for Scalable Quantum Information Processing in Diamond, Phys. Rev. X 4, 031022 (2014).

[31] N. H. Nickerson, J. F. Fitzsimons, and S. C. Benjamin, Freely Scalable Quantum Technologies Using Cells of 5-to-50 Qubits with Very Lossy and Noisy Photonic Links, Phys. Rev. X 4, 041041 (2014).

[32] A. Russo, E. Barnes, and S. E. Economou, Photonic graph state generation from quantum dots and color centers for quantum communications, Phys. Rev. B 98, 085303 (2018).

[33] R. Karhu, E. Ö. Sveinbjörnsson, B. Magnusson, I. G. Ivanov, Ö. Danielsson, and J. Ul Hassan, CVD growth and properties of on-axis vanadium-doped semi-insulating 4H-SiC epilayers, J. Appl. Phys 125, 085303 (2019).

[34] M. Kunzer, H. D. Müller, and U. Kaufmann, Magnetic circular dichroism and site-selective optically detected magnetic resonance of the deep amphoteric vanadium impurity in 6H-SiC, Phys. Rev. B 48, 10846 (1993).

[35] V. Ivády, A. Gällström, N. T. Son, E. Janzén, and A. Gali, Asymmetric Split-Vacancy Defects in SiC Polytypes: A Combined Theoretical and Electron Spin Resonance Study, Phys. Rev. Lett. 107, 195501 (2011).

[36] J. P. Perdew, K. Burke, and M. Ernzerhof, Generalized Gradient Approximation Made Simple, Phys. Rev. Lett. 77, 3865 (1996).

[37] P. E. Blöchl, Projector augmented-wave method, Phys. Rev. B 50, 17953 (1994).

[38] G. Kresse and J. Furthmüller, Efficient iterative schemes for ab initio total-energy calculations using a plane-wave basis set, Phys. Rev. B 54, 11169 (1996).

[39] A. Gali, E. Janzén, P. Deák, G. Kresse, and E. Kaxiras, Theory of Spin-Conserving Excitation of the $N-V^{-}$Center in Diamond, Phys. Rev. Lett. 103, 186404 (2009).

[40] A. Gali, T. Demján, M. Vörös, G. Thiering, E. Cannuccia, and A. Marini, Electron-vibration coupling induced renormalization in the photoemission spectrum of diamondoids, Nat. Comm. 7, 11327 (2016).

[41] A. Alkauskas, B. B. Buckley, D. D. Awschalom, and C. G. Van de Walle, First-principles theory of the luminescence lineshape for the triplet transition in diamond NV centres, New J. Phys. 16, 073026 (2014).

[42] J. Heyd, G. E. Scuseria, and M. Ernzerhof, Hybrid functionals based on a screened coulomb potential, J. Chem. Phys. 118, 8207 (2003). 
[43] P. Deák, B. Aradi, T. Frauenheim, E. Janzén, and A. Gali, Accurate defect levels obtained from the HSE06 rangeseparated hybrid functional, Phys. Rev. B 81, 153203 (2010).

[44] C. Freysoldt, B. Grabowski, T. Hickel, J. Neugebauer, G. Kresse, A. Janotti, and C. G. Van de Walle, First-principles calculations for point defects in solids, Rev. Mod. Phys. 86, 253 (2014).

[45] V. Ivády, R. Armiento, K. Szász, E. Janzén, A. Gali, and I. A. Abrikosov, Theoretical unification of hybrid-DFT and DFT $+u$ methods for the treatment of localized orbitals, Phys. Rev. B 90, 035146 (2014).

[46] S. Lany and A. Zunger, Generalized Koopmans density functional calculations reveal the deep acceptor state of $\mathrm{NO}$ in ZnO, Phys. Rev. B 81, 205209 (2010).

[47] V. Ivády, I. Abrikosov, E. Janzén, and A. Gali, Role of screening in the density functional applied to transitionmetal defects in semiconductors, Phys. Rev. B 87, 205201 (2013).

[48] W. C. Mitchel, W. D. Mitchell, G. Landis, H. E. Smith, W. Lee, and M. E. Zvanut, Vanadium donor and acceptor levels in semi-insulating 4H-and 6H-SiC, J. Appl. Phys. 101, 013707 (2007).

[49] E. Londero, G. Thiering, L. Razinkovas, A. Gali, and A. Alkauskas, Vibrational modes of negatively charged silicon-vacancy centers in diamond from ab initio calculations, Phys. Rev. B 98, 035306 (2018).

[50] B. Kaufmann, A. Dörnen, and F. S. Ham, Crystal-field model of vanadium in $6 \mathrm{H}$ silicon carbide, Phys. Rev. B 55, 13009 (1997).

[51] N. J. Stone, INDC (NDS)-0658, Tech. Rep. (IAEA Vienna, 2014).

[52] S. T. Merkel, P. S. Jessen, and I. H. Deutsch, Quantum control of the hyperfine-coupled electron and nuclear spins in alkali-metal atoms, Phys. Rev. A 78, 023404 (2008).

[53] B. E. Anderson, H. Sosa-Martinez, C. A. Riofrío, I. H. Deutsch, and P. S. Jessen, Accurate and Robust Unitary Transformations of a High-Dimensional Quantum System, Phys. Rev. Lett. 114, 240401 (2015).

[54] M. H. Metsch, K. Senkalla, B. Tratzmiller, J. Scheuer, M. Kern, J. Achard, A. Tallaire, M. B. Plenio, P. Siyushev, and F. Jelezko, Initialization and readout of nuclear spins via negatively charged silicon-vacancy center in diamond, arXiv:1902.02965 [quant-ph] (2019), http://arxiv.org/abs/1902.02965v3.

[55] G. Calusine, A. Politi, and D. D. Awschalom, CavityEnhanced Measurements of Defect Spins in Silicon Carbide, Phys. Rev. Appl. 6, 014019 (2016).

[56] D. O. Bracher, X. Zhang, and E. L. Hu, Selective Purcell enhancement of two closely linked zero-phonon transitions of a silicon carbide color center, Proc. Natl. Acad. Sci. U. S. A. 114, 4060 (2017).

[57] T.-K. Nguyen, H.-P. Phan, H. Kamble, R. Vadivelu, T. Dinh, A. Iacopi, G. Walker, L. Hold, N.-T. Nguyen, and D. V. Dao, Superior robust ultrathin single-crystalline silicon carbide membrane as a versatile platform for biological applications, ACS Appl. Mater. Interfaces 9, 41641 (2017).

[58] Y. Q. Gao, H. Y. Zhang, J. Song, S. Song, Q. R. Liang, M. Ning, C. Gao, X. J. Wang, Y. M. Zong, H. H. Wang, and M. Dudley, in Silicon Carbide and Related Materials 2014, Materials Science Forum, (Trans Tech Publications, Zürich-Stafa, Pfaffikon, 2015), Vol. 821, p. 51.
[59] J.-M. Gerard and B. Gayral, Strong Purcell effect for InAs quantum boxes in three-dimensional solid-state microcavities, J. Lightwave Technol. 17, 2089 (1999).

[60] G. W. Biedermann, F. M. Benito, K. M. Fortier, D. L. Stick, T. K. Loyd, P. D. D. Schwindt, C. Y. Nakakura, R. L. Jarecki, and M. G. Blain, Ultrasmooth microfabricated mirrors for quantum information, Appl. Phys. Lett. 97, 181110 (2010).

[61] C. Derntl, M. Schneider, J. Schalko, A. Bittner, J. Schmiedmayer, U. Schmid, and M. Trupke, Arrays of open, independently tunable microcavities, Opt. Express 22, 22111 (2014).

[62] S. Kuhn, G. Wachter, F.-F. Wieser, J. Millen, M. Schneider, J. Schalko, U. Schmid, M. Trupke, and M. Arndt, Nanoparticle detection in an open-access silicon microcavity, Appl. Phys. Lett. 111, 253107 (2017).

[63] G. Wachter, S. Kuhn, S. Minniberger, C. Salter, P. Asenbaum, J. Millen, M. Schneider, J. Schalko, U. Schmid, A. Felgner, D. Hüser, M. Arndt, and T. Michael, Silicon microcavity arrays with open access and a finesse of half a million, Light: Sci. Appl. 8, 37 (2019).

[64] M. Hanks, M. Trupke, J. Schmiedmayer, W. J. Munro, and K. Nemoto, High-fidelity spin measurement on the nitrogen-vacancy center, New J. Phys. 19, 103002 (2017).

[65] E. Janitz, M. Ruf, M. Dimock, A. Bourassa, J. Sankey, and L. Childress, Fabry-Perot microcavity for diamond-based photonics, Phys. Rev. A 92, 043844 (2015).

[66] J. Benedikter, H. Kaupp, T. Hümmer, Y. Liang, A. Bommer, C. Becher, A. Krueger, J. M. Smith, T. W. Hänsch, and D. Hunger, Cavity-Enhanced Single-Photon Source Based on the Silicon-Vacancy Center in Diamond, Phys. Rev. Appl. 7, 024031 (2017).

[67] D. Najer, M. Renggli, D. Riedel, S. Starosielec, and R. J. Warburton, Fabrication of mirror templates in silica with micron-sized radii of curvature, Appl. Phys. Lett. 110, 011101 (2017).

[68] S. Bogdanović, S. B. van Dam, C. Bonato, L. C. Coenen, A.-M. J. Zwerver, B. Hensen, M. S. Z. Liddy, T. Fink, A. Reiserer, M. Lončar, and R. Hanson, Design and lowtemperature characterization of a tunable microcavity for diamond-based quantum networks, Appl. Phys. Lett. 110, 171103 (2017).

[69] P. Senellart, G. Solomon, and A. White, High-performance semiconductor quantum-dot single-photon sources, Nature Nanotechnol. 12, 1026 (2017).

[70] S. E. Economou, N. Lindner, and T. Rudolph, Optically Generated 2-Dimensional Photonic Cluster State from Coupled Quantum Dots, Phys. Rev. Lett. 105, 093601 (2010).

[71] M. Fleischhauer, A. Imamoglu, and J. P. Marangos, Electromagnetically induced transparency: Optics in coherent media, Rev. Mod. Phys. 77, 633 (2005).

[72] O. V. Zwier, D. O'Shea, A. R. Onur, and C. H. Van Der Wal, All-optical coherent population trapping with defect spin ensembles in silicon carbide, Sci. Rep. 5, 10931 (2015).

[73] C. G. Yale, F. Joseph Heremans, B. B. Zhou, A. Auer, G. Burkard, and D. D. Awschalom, Optical manipulation of the berry phase in a solid-state spin qubit, Nat. Photonics 10, 184 (2016).

[74] A. Rickman, The commercialization of silicon photonics, Nat. Photonics 8, 579 (2014). 